\title{
Effect of Curcumin in Comparison with Trichostatin A on the Reactivation of Estrogen Receptor Alpha gene Expression, Cell Growth Inhibition and Apoptosis Induction in Hepatocellular Carcinoma Hepa 1-6 Cell ILine
}

\author{
Masumeh Sanaei ${ }^{1}$, Fraidoon Kavoosi ${ }^{1 *}$, Mehrnoosh Arabloo ${ }^{2}$
}

\begin{abstract}
Background: A multistep process with an accumulation of epigenetic alterations of tumor suppressor genes (TSGs) can induce cancer. Abnormal regional hypermethylation and histone deacetylation of several TSGs has been observed in hepatocellular carcinoma (HCC). Acetylation and deacetylation of histone are carried out by histone acetyltransferase (HAT) and histone deacetylase (HDAC) respectively. Besides, DNA methylation is carried out by DNA methyltransferases (DNMTs). Previously, we evaluated the effect of DNA demethylating agents and histone deacetylase inhibitors on HCC and colon cancer. This study aimed to evaluate the effect of curcumin (CUR) in comparison with trichostatin A (TSA) on estrogen receptor alpha (ER $)$ reactivation, apoptotic induction, and cell growth inhibition in HCC. Methods: the cells were cultured and treated with various concentrations of CUR and TSA and the MTT assay, flow cytometry assay and Real-Time RT-PCR were achieved to determine cell viability, cell apoptosis, and $E R \alpha$ gene expression respectively. Results: CUR indicated dose and time-dependent antiproliferative effects $(\mathrm{P}<0.035)$. A similar antiproliferative effect was observed by TSA $(\mathrm{P}<0.001)$. Both compounds indicated significant apoptotic effects in all different periods $(\mathrm{P}<0.001)$, CUR indicated a more significant apoptotic effect than TSA $(\mathrm{P}<0.001)$. The ER $\alpha$ gene expression quantity was increased significantly by treatment with CUR and TSA $(\mathrm{P}<0.012)$. Conclusion: CUR and TSA play important roles in restoring the ER $\alpha$ resulting in cell growth inhibition and apoptosis induction. Therefore, $\mathrm{ER} \alpha$ may be a potential target for therapeutic intervention in the treatment of HCC.
\end{abstract}

Keywords: Curcumin- Trichostatin A- Estrogen receptor alpha- Hepatocellular carcinoma

Asian Pac J Cancer Prev, 21 (4), 1045-1050

\section{Introduction}

The most primary malignancy of the liver is hepatocellular carcinoma (HCC) which is a leading cause of cancer-related death worldwide (Balogh et al., 2016). The most common risk factors of HCC are hepatitis B virus (HBV) and hepatitis $\mathrm{C}(\mathrm{HBC})$ virus infections (Zidan et al., 2012). Besides, associations between epigenetic alterations and cancer induction are well known. A multistep process with an accumulation of epigenetic alterations of tumor suppressor genes (TSGs) can induce cancer (Esteller, 2007). One of the main mechanisms of carcinogenesis is the hypermethylation of $\mathrm{CpG}$ islands in the promoter region of TSGs (Yang et al., 2017). Abnormal regional hypermethylation of the $5^{\prime}$ region of several TSGs has been observed in HCC (Baylin et al., 2011). Down-regulation of a variety of TSGs expression because of abnormal hypermethylation in their promoter regions could play a critical role in liver tumorigenesis
(Nishida et al., 2011). DNA methylation is carried out by DNA methyltransferases (DNMTs) which transfer a methyl group to the cytosine of $\mathrm{CpG}$ dinucleotides. There is a family of these enzymes in the eukaryotes with five members, including Dnmt1, Dnmt2, Dnmt3a, Dnmt3b and Dnmt3L (Li., 2002; Bestor., 2000). DNMT1 is crucial for the maintenance of DNA methylation, which is important for the initiation of chromatin remodeling and regulation of gene expression (Espada et al., 2004; Milutinovic et al., 2004). More than 600 natural compounds have been reported with a pharmaceutical activity which many of them have anti-cancer activity, one of which is curcumin (CUR), diferuloylmethane, a powerful chemo-preventive, and anti-tumor agent (Bar-Sela et al., 2010). CUR exerts its effect through epigenetic modification by targeting numerous epigenetic factors such as DNMTs, histone acetyltransferase (HAT), histone deacetylase (HDAC) and miRNAs (Reuter et al., 2011; Balasubramanyam et al., 2004). Thus, the regulatory roles of CUR include 
DNMTs inhibition, histone modulations, and miRNA regulation (Boyanapalli et al., 2015). Furthermore, histone deacetylation is associated with carcinogenesis. Acetylation and deacetylation of histone carry out by HAT and HDAC respectively. In many tumors, the balance between HAT and HDAC activity is altered (Bannister et al., 2011). Histone deacetylase inhibitors (HDACIs) play an important role in cancer prevention and cancer treatment by promoting acetylation of histones and non-histone protein substrates. They can induce apoptosis and cell cycle arrest by acetylation of histone proteins. These compounds are classified into four classes, including hydroxamic acids, short-chain fatty acids, cyclic peptides, and synthetic benzamides (Lakshmaiah et al., 2014). Significant inhibitory and apoptotic effects of hydroxamic acid trichostatin A (TSA) on HCC HepG 2 have been reported (Shi et al., 2014).

It has been reported that HDAC inhibitor TSA in combination with CUR has greater antiproliferative and apoptotic effects than either agent alone in breast cancer (Yan et al., 2013).

Estrogen receptor alpha $(E R \alpha)$ is one of the TSGs that has recently attracted the attention of researchers and its role in various cancers has been reported. It is expressed in many tissues such as the breast and liver and is a very important choice for endocrine therapy (Halon et al., 2011; Kanda et al., 2014). Previously, we demonstrated that genistein (GE) and TSA can induce apoptosis by re-activation of estrogen receptor alpha $(E R \alpha)$ in the HCC Hep G2 cell line (Sanaei et al., 2017). However, to our researches, few studies have evaluated the effect of CUR and TSA on ER $\alpha$ reactivation, apoptotic induction and cell growth inhibition of human HCC Hepa 1-6 cell. Therefore, in the current study, we investigated the comparative effects of these compounds on the ER $\alpha$ gene expression, cell growth inhibition and apoptosis induction in this cell line.

\section{Materials and Methods}

\section{Cell Lines and cell culture}

Human hepatocellular carcinoma Hepa 1-6 cells were provided from the National Cell Bank of Iran-Pasteur Institute and maintained in Dulbecco's modified Eagle's medium (DMEM) supplemented with 10\% fetal bovine serum, $1 \%$ antibiotics, including streptomycin sulfate, penicillin $\mathrm{G}$ sodium (Sigma), and amphotericin B (Sigma) at $37^{\circ} \mathrm{C}$ in $5 \% \mathrm{CO}_{2}$ to promote attachment. The Hepa 1-6 cells were cultured and treated with drugs (CUR and TSA) after they attached and reached $>80 \%$ confluence. Both agents were purchased from Sigma-Aldrich (St. Louis, MO, USA) and dissolved in dimethyl sulfoxide (DMSO) to prepare a stock solution and then all test concentrations were prepared by diluting this solution. Other compounds, including trypsin-EDTA, fetal bovine serum (FBS), and phosphate-buffered saline (PBS) were purchased from Sigma Chemical Co. (St. Louis, MO, USA). Annexin$\mathrm{V}$-(FITC) and propidium iodide (PI) were prepared from sigma (Becton-Dickinson, San Diego, CA) too.
Cell growth and cell viability assay

Using MTT assay, the effect of CUR and TSA on the cell viability was measured. First, the cells were cultured into 96-well plates (at a density of $4 \times 10^{5}$ cells per well) and allowed to adhere overnight. After $24 \mathrm{~h}$ of culture, the culture medium was removed and a medium containing different doses of CUR $(0.5,1,5,10$ and $25 \mu \mathrm{M})$ and TSA $(0.5,1,5,10$ and $25 \mu \mathrm{M})$ were added, except control groups incubated with DMSO only. After treatment times ( 24 and $48 \mathrm{~h}$ ), the treated cells were washed twice with FBS, maintained in a medium containing MTT for $4 \mathrm{~h}$ and then the formazan crystals were dissolved in DMSO and the absorbance was measured at $570 \mathrm{~nm}$. All experiments were repeated three times.

\section{Cell apoptosis assay}

For apoptotic effect determination, the Hepa 1-6 cells were cultured in 24-well plates at a density of $4 \times 10^{5}$ cells/well and incubated overnight before treating with drugs. After $24 \mathrm{~h}$, the cells were treated with CUR (5 $\mu \mathrm{M})$ and TSA $(1 \mu \mathrm{M})$, Based on $\mathrm{IC}_{50}$ values, for different periods ( 24 and $48 \mathrm{~h}$ ). After treatment times, the culture medium was removed and all the adherent cells were harvested by trypsinization, washed with cold PBS, and then the cells resuspended in Binding buffer (1x). Finally, Annexin-V-(FITC) and PI were used to stain the cells according to the protocol and the apoptotic cells were counted by FACScan flow cytometry (Becton Dickinson, Heidelberg, Germany).

\section{Real-time Quantitative Reverse Transcription Polymerase Chain Reaction ( $q R T-P C R$ )}

To determine whether CUR and TSA could reactivate the ER $\alpha$ gene expression, qRT-PCR was performed. In this regard, the cells were cultured and treated with CUR and TSA ( 5 and $1 \mu \mathrm{M}$ respectively, based on $\mathrm{IC}_{50}$ values) for 24 and $48 \mathrm{~h}$ and total RNA from the cells was extracted using the RNeasy kit (Qiagen, Valencia, CA) according to the protocol and treated by RNase-free DNase (Qiagen) to eliminate the genomic DNA before cDNA synthesis. The concentration of RNA was determined using a BioPhotometer (Biowave II Germany). By using the RevertAid $^{\text {TM }}$ First Strand cDNA Synthesis Kit (Fermentas, K1622 for 100 reactions), total RNA(100 ng) was reversetranscribed to complementary DNA (cDNA) according to the protocol.

Real-time RT-PCR was performed by the Maxima SYBR Green RoxqPCR master mix kit (Fermentas). ER $\alpha$ primers were obtained from our previous work (Kim S-H et al., 2010) which their sequences are shown in Table1. GAPDH was used as an endogenous control.

Data were analyzed using the comparative $\mathrm{Ct}$ ( $\Delta \Delta \mathrm{ct}$ ) method, the relative expression level of $E R \alpha$ was calculated by determining a ratio between the amount of the gene and that of endogenous control.

\section{Results}

Effect of CUR and TSA on cell viability of Hepa 1-6 cells

The Hepa 1-6 cells were seeded in 96-well plates (at a density of $4 \times 10^{5}$ per well) and treated with CUR 
Table 1. Primer Sequence Used in the Study

\begin{tabular}{ll}
\hline Genes & Primer sequences \\
\hline ER $\alpha$ & \\
Forward: & 5'-AGA CAT GAG AGC TGC CAA CC-3' \\
Reverse: & 5'-GCC AGG CAC ATT CTA GAA GG-3' \\
\hline
\end{tabular}

Table 2. Percentage of Apoptosis in the Groups Treated with CUR and TSA at Different Periods

\begin{tabular}{ccccc}
\hline Drug & Dose $/ \mu \mathrm{M}$ & Duration $/ \mathrm{h}$ & Apoptosis $\%$ & $P$-value \\
\hline CUR & 5 & 24 & 68.47 & $\mathrm{P}<0.001$ \\
& 5 & 48 & 96.10 & $\mathrm{P}<0.001$ \\
TSA & 1 & 24 & 20.10 & $\mathrm{P}<0.001$ \\
& 1 & 48 & 24.96 & $\mathrm{P}<0.001$ \\
\hline
\end{tabular}

$(0.5,1,5,10$ and $25 \mu \mathrm{M})$ and TSA $(0.5,1,5,10$ and 25 $\mu \mathrm{M}$ ) for 1 and 2 days as mentioned above and then the cell viability was determined by MTT assay. As shown in Figure 1, CUR indicated dose- and time-dependent significant antiproliferative effects $(\mathrm{P}<0.001)$. A similar antiproliferative effect was observed by TSA treatment as shown in Figure 1. $(\mathrm{P}<0.002)$. The result of MTT assay demonstrated that CUR and TSA inhibited cell growth with an $\mathrm{IC}_{50}$ of $\sim 5$ and $\sim 1 \mu \mathrm{M}$ respectively.

\section{Effect of CUR and TSA on cell apoptosis}

For determination of the apoptotic effects of CUR and TSA on Hepa 1-6, the cells were treated with CUR $(5 \mathrm{M} \mu)$ and TSA $(1 \mathrm{M} \mu)$ for 24 and $48 \mathrm{~h}$ and then the apoptotic effects of these compounds were determined by flow cytometry assay. The result of flow cytometry showed that both compounds indicated significant apoptotic effects in all different periods as shown in Figure $2(\mathrm{P}<0.001)$. As seen in Figure 2, CUR indicated a more significant apoptotic effect than TSA $(\mathrm{P}<0.001)$. The percentage of apoptotic cells is shown in Table 2 .

\section{Effect of CUR and TSA on ERa gene expression}

To determine the mechanism of CUR $(5 \mathrm{M} \mu)$ and TSA
Table 3. Relative Expression Level of ERa Gene Expression

\begin{tabular}{lccccc}
\hline Gene & Drug & $\begin{array}{c}\text { Dose } \\
(\mu \mathrm{M})\end{array}$ & $\begin{array}{c}\text { Duration } \\
(\mathrm{h})\end{array}$ & Expression & $\begin{array}{c}P- \\
\text { value }\end{array}$ \\
\hline$E R \alpha$ & TSA & 1 & 24 & 1.6 & 0.020 \\
$E R \alpha$ & TSA & 1 & 48 & 2.4 & 0.001 \\
$E R \alpha$ & CUR & 5 & 24 & 2.1 & 0.001 \\
$E R \alpha$ & CUR & 5 & 48 & 2.8 & 0.001 \\
\hline
\end{tabular}

(1 $\mathrm{M} \mu)$ on ER $\alpha$ gene expression, RT-PCR was done to test the $E R \alpha$ gene expression quantity during treatment with these agents at different periods. As shown in Figure 3 and Table 3, the ER $\alpha$ gene expression quantity was increased significantly by treatment with CUR and TSA $(\mathrm{P}<0.012)$. The effect of CUR was more significant than that of the TSA.

\section{Discussion}

Cancer cells are activated by genetic and epigenetic alterations, epigenetic processes such as hypermethylation and deacetylation of TSGs. These processes silence TSGs resulting in cancer induction. Hypermethylation creates a closed chromatin conformation that affects gene expression and involves cellular processes such as cell cycle, cell growth, cell differentiation, and cell apoptosis. Numerous medicinal plant-derived compounds have been reported to modulate the activation of diverse silenced genes in various cancers. Curcumin is a well-known anti-oxidative and anti-inflammatory agent which prevents several cancers via epigenetic regulation, including DNMTs inhibition, histone modifications, and microRNAs regulation (Boyanapalli et al., 2015). HDACs are also critical regulators of gene expression. Acetylation and deacetylation of histone proteins are achieved by two groups of enzymes, including HATs and HDACs. Histone deacetylation has been related to a broad range of cancer types. HADIs play an important role in cancer prevention and treatment. Histone deacetylase inhibitor

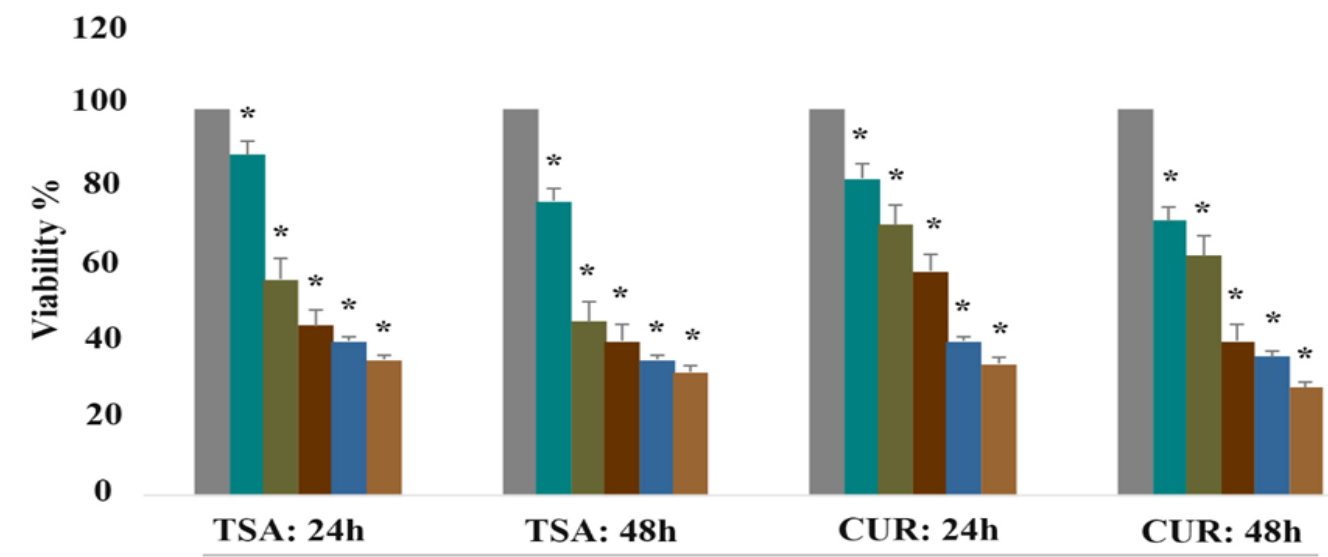

Concentrations: $0.5,1,5,10$ and $25 \mu \mathrm{M}$

Figure 1. The Effect of CUR $(0.5,1,5,10$ and $25 \mu \mathrm{M})$ and TSA $(0.5,1,5,10$ and $25 \mu \mathrm{M})$ on the Hepa 1-6 Cell Viability. The effects were determined by the MTT assay. Data are presented as mean \pm SD from at least triplicate wells and 3 independent experiments. Significant differences between treated cells and the control group are indicated by asterisks $(*)$. The first column of each group belongs to the control group 

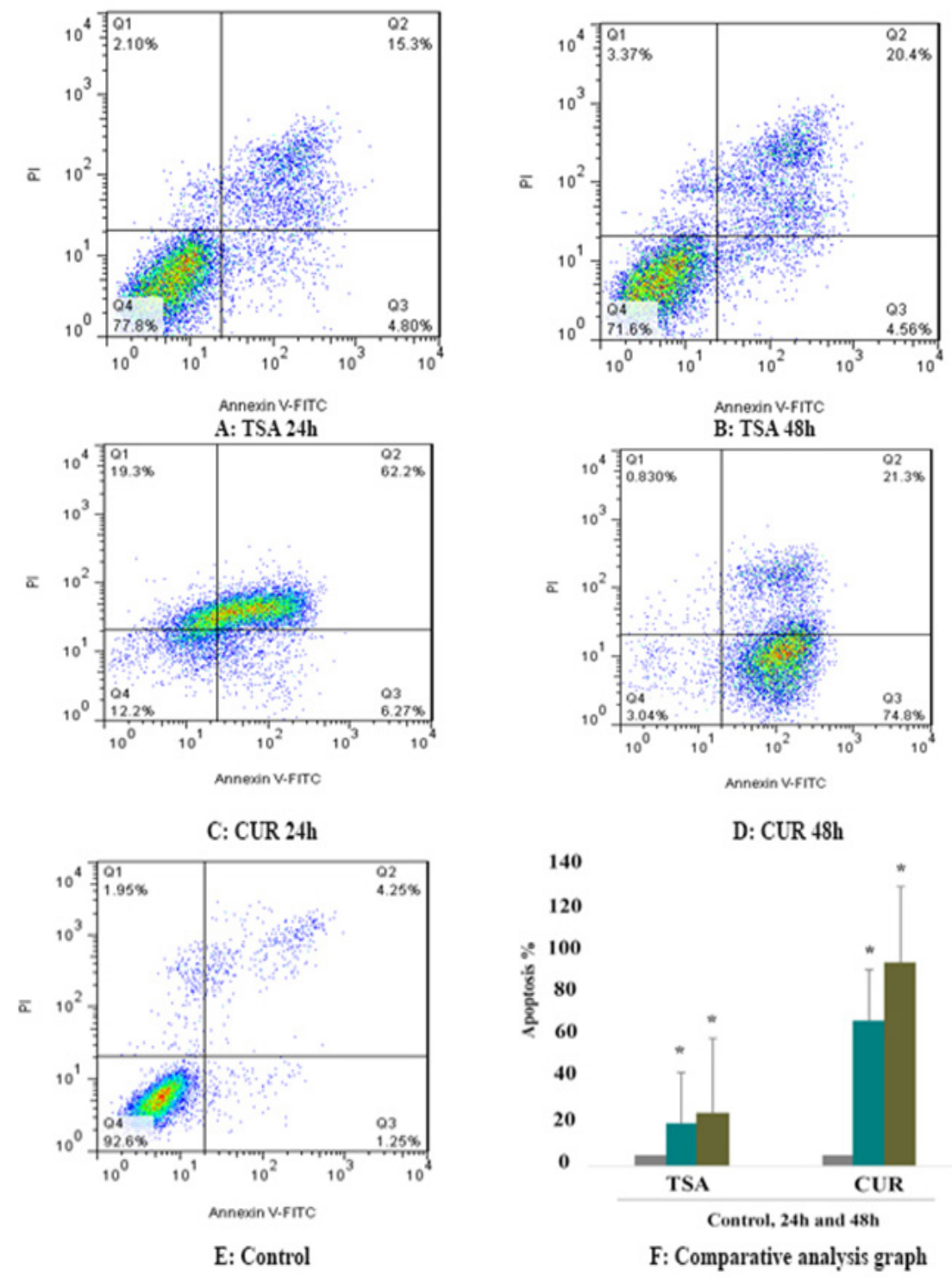

Figure 2. Apoptotic Effects of CUR and TSA on Hepa 1-6 Cell Line. Significant apoptosis was shown at different periods in a time-dependent manner $(P<0.001)$.

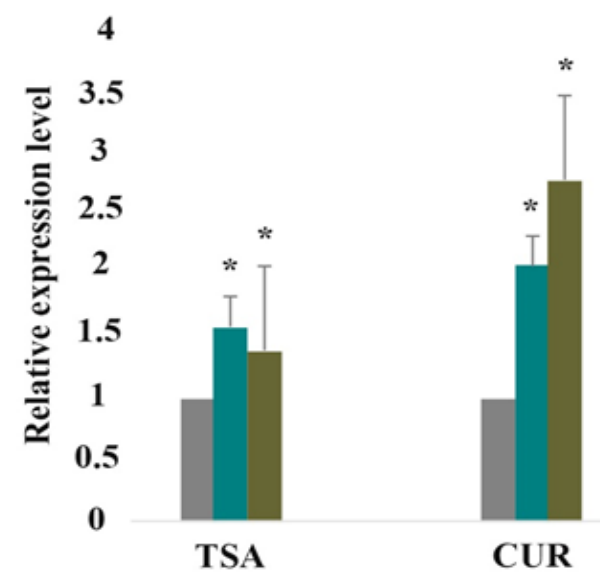

Figure 3. The Relative Expression Level of ER $\alpha$. As can bee seen above, CUR and TSA increased ER $\alpha$ gene expression significantly. Data are presented as mean \pm standard error of the mean from at least three different experiments. It should be noted that the effect of CUR was stronger than that of the TSA. Asterisks $(*)$ indicate significant differences between treated cells and the control group.
TSA can restore silenced TSGs such as ER $\alpha$ by acetylation of these genes (Kim et al., 2010). In the present study, we showed that CUR and TSA can epigenetically inhibit cell growth and induce apoptosis in Hepa 1-6 cells and that CUR induces a more significant apoptotic effect than TSA. Besides, our work indicated that both compounds increase $E R \alpha$ gene expression significantly. The effect of CUR on $E R \alpha$ gene expression was more significant than the TSA.

Similar to our report, it has been shown that TSA can suppress the proliferation of primary hepatic cells and breast cancer cells by acetylation of histone (Kim et al., 2010; Xiong et al., 2012; Yang et al., 2000). There are many studies that have been reported that TSA inhibits growth and induces apoptosis in other cancers, including ovarian cancer, lung cancer epithelial-like cell (SKOV-3 and A549 respectively), urinary bladder cancer cells and esophageal squamous cell carcinoma (Hassan et al., 2014; Wang et al., 2017; Ma et al., 2015). In line with our result, it has been reported that CUR inhibits proliferation and induces apoptosis in HCC J5 cells (Cheng et al., 2008). The most evaluated component combinations are those 
that affect histone deacetylation (such as HDACIs) and DNA methylation (such as DNMTIs) (Ahuja et al., 2016; Azad et al., 2013; Egger et al., 2004).

One of the mechanisms by which CUR exerts its mechanism is through epigenetic factors such as HDAC. Several studies have reported that CUR modulates various molecular pathways, including cell cycle proteins (e.g., cyclin D1) and apoptosis-related proteins (e.g., Bcl-2, caspases, DR, Fas) (Shishodia., 2013). CUR exerts an antiproliferative effect through DNMT1, it blocks the catalytic thiolate of C1226 of DNMT1 to exert its antiproliferative effect (Liu et al., 2009). Other signaling events inhibited by CUR include Jun/Ap-1 activation, phosphorylations catalyzed by protein kinases and prostaglandin biosynthesis (Nakamura et al., 2002; Shehzad et al., 2010; Koeberle et al., 2009). Our previous study indicated that TSA, as a histone deacetylase inhibitor, can up-regulate $E R \alpha$ gene expression and induce apoptosis in Hep G2 HCC (Sanaei et al., 2017). In the current study, we demonstrated that TSA induces apoptosis by the up-regulation of ER $\alpha$. Similarly, this pathway has been reported for TSA in breast cancer (Stark et alo., 2013). Drug-induced accumulation of acetylated proteins such as p53, BCL6, and Hsp90 is one of the other mechanisms of suberoylanilide hydroxamic acid (SAHA) (Richon., 2006). The molecular mechanism of the apoptotic effect of SAHA in chronic myelocytic leukemia $\mathrm{BV}-173$ cells is Bcr-Abl and c-Myc downregulation (Xu et al., 2005). It has been reported that TSA can activate certain hypermethylated genes, including $C D K N 2 B$, CDKN2A, MLH1 and TIMP3 in colon cancer cells after Dnmt1 inhibition by DNA demethylating agent such as 5-aza-dc (Cameron et al., 1999). which may support this thesis that CUR increases ER $\alpha$ gene expression by $D N M T 1$ gene down-regulation. All reports that mentioned above are inconsistent with our results. Significant downregulation of $E R \alpha$ expression has been reported in breast cancer that is opposite to our result (Nejati-Koshki et al., 2014). Additionally, an in vitro study has reported that curcumin can inhibit colon cancer HCT-116 cell line as a dose dependent manner (Khameneh ZR et al., 2018). With regard to our report about the effects of $C U R$ and $T S A$ on ER $\alpha$ gene expression and other reports about the effect of CUR on DNMT1 inhibition, investigation of the effects of these compounds on DNMT1 expression is recommended.

In conclusion, collectively, our result suggests an important role of CUR and TSA on ER $\alpha$ re-activation, cell growth inhibition and apoptosis induction of hepatocellular carcinoma, which may provide a new preventive and therapeutic strategy for cancer treatment.

\section{Acknowledgments}

We thank the adjutancy of research of Jahrom Medical University and members of our groups for helpful comments and discussion. The article has been extracted from Ms. Mehrnoosh Arabloo's thesis.

\section{Funding}

This work was supported by the adjutancy of research of Jahrom medical University-Iran. This article has been extracted from Ms. Mehrnoosh Arabloo's thesis.

\section{Conflict of interest}

The authors report no conflict of interest.

\section{References}

Ahuja N, Sharma AR, Baylin SB (2016). Epigenetic therapeutics: a new weapon in the war against cancer. Annu Rev Med, 67, 73-89.

Azad N, Zahnow CA, Rudin CM, et al (2013). The future of epigenetic therapy in solid tumours - lessons from the past. Nat Rev Clin Oncol, 10, 256-66.

Balasubramanyam K, Varier RA, Altaf M, et al (2004). Curcumin, a novel p300/CREB-binding protein-specific inhibitor of acetyltransferase, represses the acetylation of histone/nonhistone proteins and histone acetyltransferasedependent chromatin transcription. J Biol Chem, 279, 51163-71.

Balogh J, David Victor III EHA, Burroughs SG, et al (2016). Hepatocellular carcinoma: a review. $J$ Hepatocellular Carcinoma, 3, 41-53.

Bannister AJ, Kouzarides T (2011). Regulation of chromatin by histone modifications. Cell Res, 21, 381-95.

Bar-Sela G, Epelbaum R, Schaffer M (2010). Curcumin as an anti-cancer agent: review of the gap between basic and clinical applications. Curr Med Chem, 17, 190-7.

Baylin SB, Jones PA (2011). A decade of exploring the cancer epigenome-biological and translational implications. Nat Rev Cancer, 11, 726-34.

Bestor TH (2000). The DNA methyltransferases of mammals. Hum Mol Genet, 9, 2395-402.

Boyanapalli SS, Kong A-NT (2015). Curcumin, the king of spices: epigenetic regulatory mechanisms in the prevention of cancer, neurological, and inflammatory diseases. Curr Pharmacol Rep, 1, 129-39.

Cameron EE, Bachman KE, Myöhänen S, et al (1999). Synergy of demethylation and histone deacetylase inhibition in the reexpression of genes silenced in cancer. Nat Genet, 21, 103-7.

Cheng C-Y, Lin Y-H, Su C-C (2010). Curcumin inhibits the proliferation of human hepatocellular carcinoma $\mathrm{J} 5$ cells by inducing endoplasmic reticulum stress and mitochondrial dysfunction. Int J Mol Med, 26, 673-678.

Egger G, Liang G, Aparicio A, et al (2004). Epigenetics in human disease and prospects for epigenetic therapy. Nature, 429, 457-563.

Espada J, Ballestar E, Fraga MF, et al (2004). Human DNA methyltransferase 1 is required for maintenance of the histone H3 modification pattern. J Biol Chem, 279, 37175-84.

Esteller M (2007). Cancer epigenomics: DNA methylomes and histone-modification maps. Nat Rev Genet, 8, 286-298.

Halon A, Materna V, Drag-Zalesinska M, et al (2011). Estrogen receptor alpha expression in ovarian cancer predicts longer overall survival. Pathol Oncol Res, 17, 511-9.

Hassan D, Nosratollah Z (2014). Trichostatin A-induced Apoptosis is mediated by Krüppel-like factor 4 in ovarian and lung cancer. Asian Pac J Cancer Prev, 15, 6581-6.

Kanda T, Jiang X, Yokosuka O (2014). Androgen receptor signaling in hepatocellular carcinoma and pancreatic cancers. World J Gastroenterol, 20, 9229-36.

Kim S-H, Kang H-J, Na H, et al (2010). Trichostatin A enhances acetylation as well as protein stability of ER $\alpha$ through induction of p300 protein. Breast Cancer Res, 12, 22-30.

Koeberle A, Northoff H, Werz O (2009). Curcumin blocks prostaglandin E2 biosynthesis through direct inhibition of the microsomal prostaglandin E2 synthase-1. Mol Cancer Asian Pacific Journal of Cancer Prevention, Vol $21 \quad 1049$ 
Ther, 8, 2348-55.

Lakshmaiah K, Jacob LA, Aparna S, et al (2014). Epigenetic therapy of cancer with histone deacetylase inhibitors. $J$ Cancer Res Ther, 10, 469-78.

Li E (2002). Chromatin modification and epigenetic reprogramming in mammalian development. Nat Rev Genet, 3, 662-73.

Liu Z, Xie Z, Jones W, et al (2009). Curcumin is a potent DNA hypomethylation agent. Bioorg Med Chem Lett, 19, 706-9.

Ma J, Guo X, Zhang S, et al (2015). Trichostatin A, a histone deacetylase inhibitor, suppresses proliferation and promotes apoptosis of esophageal squamous cell lines. Mol Med Rep, 11, 4525-31.

Milutinovic S, Brown SE, Zhuang Q, et al (2004). DNA methyltransferase 1 knock down induces gene expression by a mechanism independent of DNA methylation and histone deacetylation. J Biol Chem, 279, 27915-27.

Nakamura K, Yasunaga Y, Segawa T, et al (2002). Curcumin down-regulates AR gene expression and activation in prostate cancer cell lines. Int J Oncol, 21, 825-30.

Nejati-Koshki K, Akbarzadeh A, Pourhassan-Moghaddam M (2014). Curcumin inhibits leptin gene expression and secretion in breast cancer cells by estrogen receptors. Cancer Cell Int, 14, 66-73.

Nishida N, Goel A (2011). Genetic and epigenetic signatures in human hepatocellular carcinoma: a systematic review. Curr Genomics, 12, 130-7.

Reuter S, Gupta SC, Park B, et al (2011). Epigenetic changes induced by curcumin and other natural compounds. Genes Nutr, 6, 93-108.

Richon V (2006). Cancer biology: mechanism of antitumour action of vorinostat (suberoylanilide hydroxamic acid), a novel histone deacetylase inhibitor. Br J Cancer, 95, 2-6.

Sanaei M, Kavoosi F, Salehi H (2017). Genistein and trichostatin a induction of estrogen receptor alpha gene expression, Apoptosis and cell growth inhibition in hepatocellular carcinoma HepG 2 Cells. Asian Pac J Cancer Prev, 18, 3445-50.

Shehzad A, Wahid F, Lee YS (2010). Curcumin in cancer chemoprevention: molecular targets, pharmacokinetics, bioavailability, and clinical trials. Arch Pharm, 343, 489-99.

Shi Q-Q, Zuo G-W, Feng Z-Q, et al (2014). Effect of trichostatin A on anti HepG2 liver carcinoma cells: Inhibition of HDAC activity and activation of $\mathrm{Wnt} / \beta$-Catenin signaling. Asian Pac J Cancer Prev, 15, 7849-55.

Shishodia S (2013). Molecular mechanisms of curcumin action: gene expression. Bio Factors, 39, 37-55.

Stark K, Burger A, Wu J, et al (2013). Reactivation of estrogen receptor $\alpha$ by vorinostat sensitizes mesenchymal-like triplenegative breast cancer to aminoflavone, a ligand of the aryl hydrocarbon receptor. PLoS One, 8, 74525-39.

Wang S-C, Wang S-T, Liu H-T, et al (2017). Trichostatin A induces bladder cancer cell death via intrinsic apoptosis at the early phase and Sp1-survivin downregulation at the late phase of treatment. Oncol Rep, 38, 1587-96.

Xiong H, Du W, Zhang YJ, et al (2012). Trichostatin A, a histone deacetylase inhibitor, suppresses JAK2/STAT3 signaling via inducing the promoter-associated histone acetylation of SOCS1 and SOCS3 in human colorectal cancer cells. Mol Carcinog, 51, 174-84.

Xu Y, Voelter-Mahlknecht S, Mahlknecht U (2005). The histone deacetylase inhibitor suberoylanilide hydroxamic acid down-regulates expression levels of Bcr-abl, c-Myc and $\mathrm{HDAC} 3$ in chronic myeloid leukemia cell lines. Int $\mathrm{J} \mathrm{Mol}$ Med, 15, 169-72.

Yan G, Graham K, Lanza-Jacoby S (2013). Curcumin enhances the anticancer effects of trichostatin a in breast cancer cells.
Mol Carcinog, 52, 404-11.

Yang X, Ferguson AT, Nass SJ, et al (2000). Transcriptional activation of estrogen receptor $\alpha$ in human breast cancer cells by histone deacetylase inhibition. Cancer Res, 60, 6890-4.

Yang Y, Zhao L, Huang B, et al (2017). A new approach to evaluating Aberrant DNA Methylation profiles in hepatocellular carcinoma as potential biomarkers. Sci Rep, 18, 46533-45.

Zidan A, Scheuerlein H, Schüle S, et al (2012). Epidemiological pattern of hepatitis $\mathrm{B}$ and hepatitis $\mathrm{C}$ as etiological agents for hepatocellular carcinoma in iran and worldwide. Hepat Mon, 12, 6894-7002.

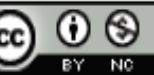

This work is licensed under a Creative Commons AttributionNon Commercial 4.0 International License. 